\title{
Labor division in joint tasks: Humans maximize use of their individual attentional capacities
}

\author{
Basil Wahn $^{1} \cdot$ Alan Kingstone $^{1}$
}

Published online: 20 May 2020

(C) The Psychonomic Society, Inc. 2020

\begin{abstract}
In daily life, humans frequently perform visuospatial tasks together (e.g., visual search) and distribute the labor in such tasks. Previous research has shown that humans prefer a left and right labor division in a joint multiple object tracking (MOT) task. Yet, findings from studies investigating individuals' tracking ability suggest attentional capacities may be more maximally used with a top and bottom labor division. We investigated whether co-actors' labor division preference is influenced by how they are seated (neighboring vs. opposite of each other) or how the MOT task is displayed (portrait vs. landscape). We find that pairs attain a higher performance using a top and bottom labor division and preferred this labor division compared to a left and right division. This preference was unaffected by the seating arrangement. For the landscape display, however, we find that participants no longer attain a higher performance for the top and bottom labor division and accordingly participants' preference for this labor division was greatly reduced as well. Overall, we propose that co-actors are sensitive to changes within their environment, which allows them to choose a labor division that maximizes use of their individual attentional capacities.
\end{abstract}

Keywords Visual attention $\cdot$ Multiple object tracking $\cdot$ Joint action $\cdot$ Social cognition $\cdot$ Task division

\section{Introduction}

In daily life, humans are bombarded with information. Yet, attentional capacity to process this information is limited (James, 1890; Chun et al., 2011; Marois \& Ivanoff, 2005). A task that has often been used to investigate attentional limitations (among several others, for reviews see: Lavie (2005) Wahn \& König, (2017a, b) is the multiple object tracking ("MOT") task (Cavanagh \& Alvarez, 2005; Pylyshyn \& Storm, 1988; Scholl, 2009). In this task, participants are required to track the movements of a varying number of objects ("targets") among moving distractor objects on a computer screen. Earlier research on this task has shown that the ability to track targets is limited (Alvarez \& Franconeri, 2007; Alnæs et al., 2014; Wahn et al., 2016a). That is, the accuracy with which participants can track target objects decreases with an increasing number of tracked targets, suggesting a limited attentional capacity.

Basil Wahn

basilwahn@gmail.com

1 Department of Psychology, University of British Columbia, Vancouver, BC, Canada
While limitations of attentional capacity have been demonstrated within the MOT task, researchers have shown that these limits are not fixed but actually change depending on how stimuli are presented in the visual field. In an earlier study (Alvarez \& Cavanagh, 2005), it was varied whether targets in the MOT task are tracked only in one visual field (e.g., only the left visual field) or in both visual fields. In particular, participants were required to track four targets in one visual field compared to tracking the same number of targets across visual fields (i.e., tracking two targets in the left visual field and two in the right visual field). Alvarez and Cavanagh (2005) found that participants reached a significantly higher tracking performance when tracking targets across visual fields compared to only one visual field, suggesting that attentional capacity is larger when performing the MOT task in both visual fields compared to only one visual field. This effect has been replicated (Chen et al., 2013) and attributed to within-Hemifield competition in early visual areas in the brain (Störmer et al., 2014).

In a recent study (Wahn et al., 2017), we extended the MOT task to a collaborative version involving pairs of participants (for a review on collaborative visuospatial tasks, see Wahn et al. (2018b)). Each member of the pair viewed the MOT stimuli on their own computer screen 
and made their own target selections. Depending on the condition, they either received performance feedback and/or information as to which targets were selected by the other participant. We found that participants devised division of labor strategies for all types of received information. Importantly, the most frequent strategy that was used was a left and right division of labor strategy. In this strategy, one participant in a pair tracked the leftmost targets while the other participant tracked the rightmost targets.

Given the findings discussed above by Alvarez and Cavanagh (2005) on individual object tracking performance, tracking targets primarily only in one visual field most likely fails to make optimal use of the individual attentional capacities of each member in the dyad. That is, as noted above, humans have a larger attentional capacity when they track targets in both visual fields compared to only in one of them. In a left and right labor division, however, task demands are divided horizontally such that each co-actor tracks her/his respective set of targets mostly in either the left or right visual field. From the perspective of the findings by Alvarez and Cavanagh (2005), a labor division for pairs that would use individual members' attentional capacities more optimally would be a top and bottom labor division. That is, if each co-actor tracked her/his set of target objects across both visual fields, and co-actors divided the labor into upper and lower halves, then pair members would use a labor division that takes advantage of each individual's higher attentional capacity for tracking targets across visual fields.

Given that a top and bottom labor division appears to make more optimal use of the members' individual attentional capacities, why did pair members in our earlier study primarily use a left and right labor division? In recent years, researchers have argued that mechanisms of coordination (or more generally, social cognition) (for reviews, see Sebanz et al. (2006); Vesper et al. (2017) can only be fully understood if the environment, in which tasks are performed, is taken into account (Goldman \& de Vignemont, 2009; Meier et al., 2012; Smith \& Semin, 2007). Hence, we hypothesized that two factors in the experimental environment may have biased participants towards using a left and right labor division in our earlier work. In particular, the participants' seating positions in our earlier study presented a clear separation into a left and right position (i.e., participants sat next to each other in front of their own computer screens and were concealed from one another by a divider), potentially biasing the participant sitting on the left to track the leftmost targets and the participant sitting on the right to track the rightmost targets. Second, we displayed the MOT task on a landscape display, potentially biasing participants towards a left and right labor division due to the wider width than height in the display. In the present study, we aimed to replicate earlier findings by Alvarez and Cavanagh (2005) and to test whether the seating arrangement (sitting opposite vs. next to each other-Experiment 1) or the screen shape (landscape vs. portrait-Experiment 2) affected participants' labor division choice.

\section{Materials and methods}

\section{Participants}

64 students of the University of British Columbia participated in the present study ( 41 females, 23 males, $M=20.91$ years, $S D=4.28$ years), which were randomly grouped in 32 pairs (16 for Experiments 1 and 2, respectively). The study was approved by the Behavioural Research Ethics Board and we obtained written consent from all participants. Students received course credits for participation.

\section{Experimental setup}

For both experiments, each participant in a pair sat in front of a separate computer screen at a distance of $60 \mathrm{~cm}$. A keyboard and mouse for making responses were placed in front of them. In Experiment 1, participants either sat opposite each other or next to each other. In Experiment 2, participants always sat opposite of each other. In all cases, participants' computer screens were concealed from each other by a divider.

\section{Experimental procedure}

In both experiments, pairs of participants performed a MOT task similar to the one by Alvarez and Cavanagh (2005). That is, for the MOT task, the screen was subdivided into four quadrants and each quadrant contained four objects (two "target" objects and two "distractor" objects). For every trial, the labor was divided by having each co-actor perform the MOT task in two quadrants that did not overlap with the quadrants of the other co-actor. As a withinsubject factor, we varied whether this labor division was pre-determined ("assigned") or could be selected by the participants ("selected"). Prior to the start of the experiment, each co-actor was randomly assigned one color (either green or yellow) to indicate in which quadrants the MOT task was to be performed in the assigned condition and to indicate the chosen quadrants in the selected condition. Below, we will explain these conditions in turn.

In the assigned condition, for each trial, co-actors first saw one out of four possible colored quadrant combinations (see Fig. 1a), each showing two quadrants colored in green and yellow, respectively. Which combination is displayed was randomly selected. The quadrant combinations either 


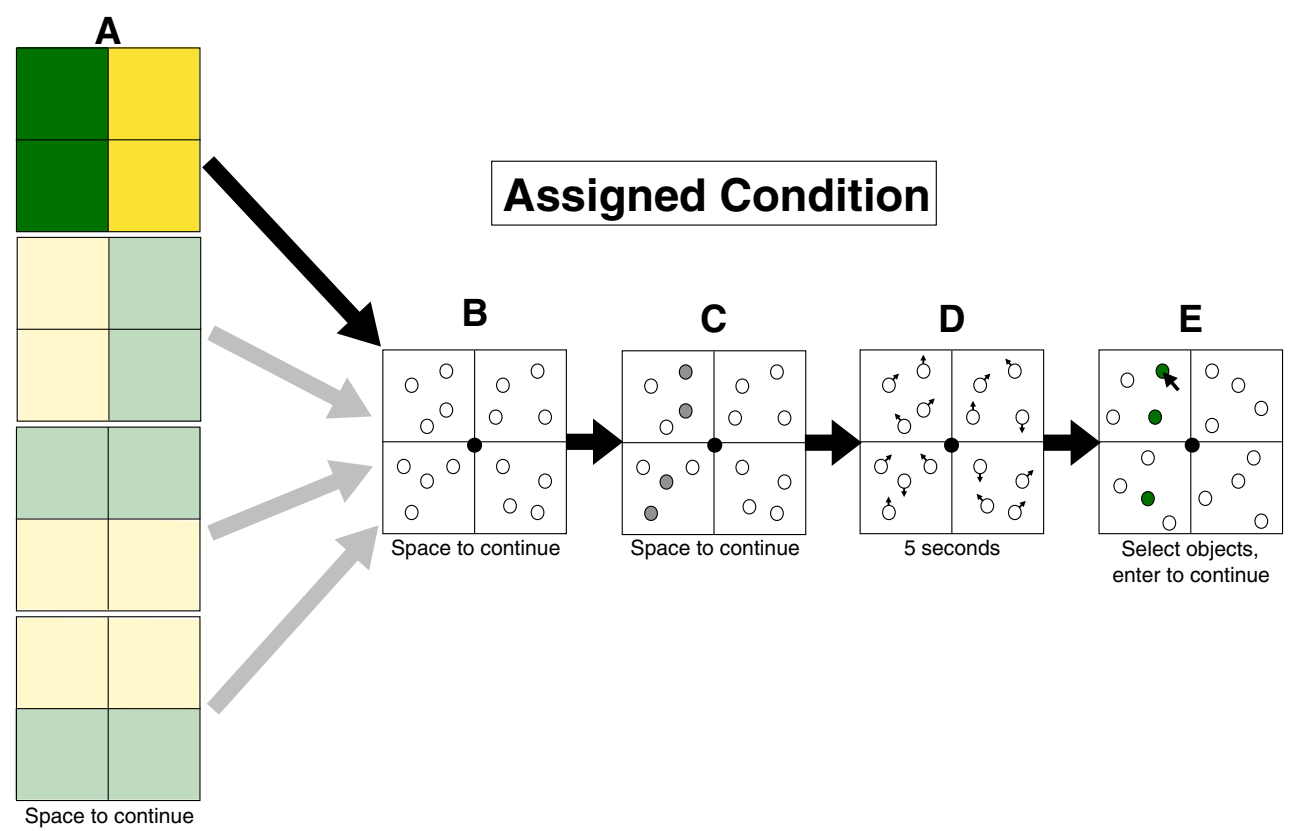

Fig. 1 Trial logic overview: Assigned condition. In this example trial, a left and right labor division is displayed from the perspective of the participant, to which the green color was assigned

displayed a left and right labor division (see A Fig. 1, upper two boxes) or a top and bottom labor division (see A Fig. 1, lower two boxes). Both participants were required to press the space key to confirm viewing the quadrant combination to proceed to the next screen.

In the selected condition, participants could choose the quadrants that they would track the targets in (see A in Fig. 2). In particular, for each trial, one of the participants was randomly selected to choose two quadrants by clicking on them with the computer mouse. Once a quadrant was selected, it changed color to the participant's assigned color. When two quadrants were chosen and the participant confirmed her/his choice by pressing the space key, the quadrant choice was displayed to the other participant. Then the other participant selected the two remaining quadrants and confirmed her/his selection by pressing the space key as well.

With the assigned condition, we aimed to replicate earlier findings by Alvarez and Cavanagh (2005). In particular, if the findings replicate, then the top and bottom labor division should yield a higher tracking performance compared to the left and right labor division as participants track targets across visual fields for the top and bottom division and only within one visual field for the left and right division. With the selected condition, we aimed to investigate participants labor division preference.

The next screens in a trial were the same for the assigned and selected conditions. Participants first saw sixteen stationary white objects ( 0.40 visual degrees radius) on the computer screen (B in Figs. 1 and 2). Then, a subset

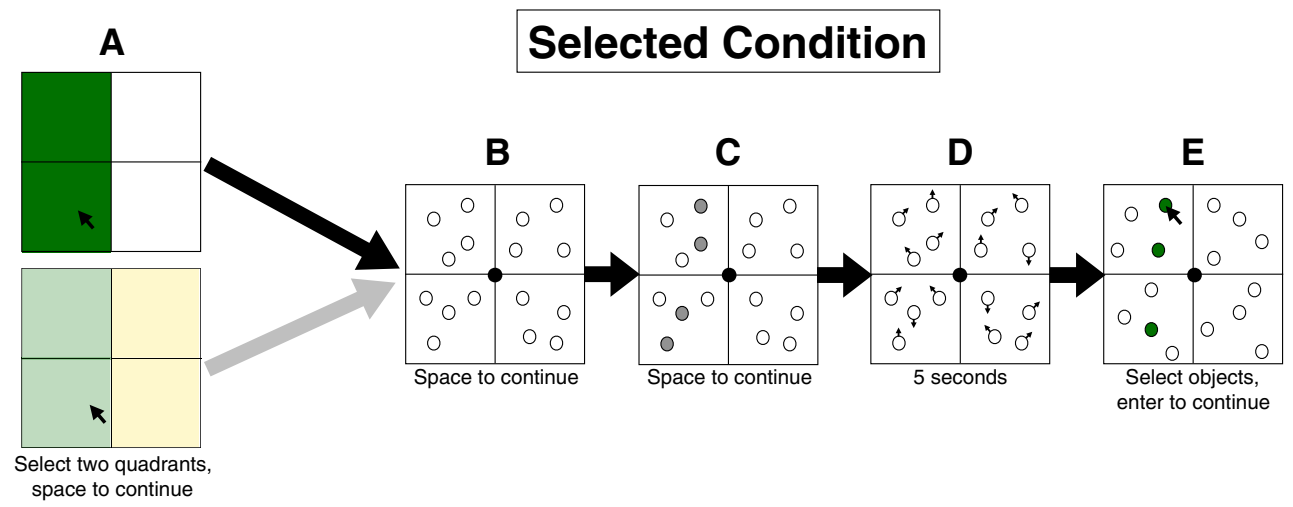

Fig. 2 Trial logic overview: Selected condition. In this example trial, the participant, to which the green color was assigned, selected two quadrants first and the rest of the trial is shown from this participant's perspective 
of four objects turned grey ("targets")—-two in each of the two previously indicated (or selected) quadrants ( $\mathrm{C}$ in Figs. 1 and 2). Then the targets reverted to looking white again and started to move in randomly chosen directions across the screen (D in Figs. 1 and 2). The object's speed varied between 1.60 and 2.40 visual degrees (per second). While objects were moving, participants were required to fixate at the central fixation dot ( 0.20 visual degrees radius). After five seconds, objects stopped moving and participants were required to select with the computer mouse those objects that they thought were the targets (E in Figs. 1 and 2). Participants were instructed to select as many objects as they wanted and to prioritize accuracy over speed with their selections. They were also informed that each correct selection would add one point to their performance and each incorrect selection would subtract one point from their performance. The entire task was self-paced. That is, participants were required to press the spacebar to proceed to the next screen (i.e., object presentation, target presentation, and starting the object movements). Yet, to avoid that participants accidentally pressed the spacebar even though they had not selected any objects, participants were required to press the enter key to confirm their targets selections. Once both participants pressed enter, a trial was complete and we displayed text on the computer screen, noting that participants should press the space key when they were ready for the next trial. Once both participants pressed the space key, the next trial would start.

Across experiments, we varied the seating position of participants (Experiment 1) or the shape of the stimuli display (Experiment 2) (see Fig. 3). In Experiment 1, participants either sat next to each other ("Neighboring" condition) or opposite of each other ("Opposite" condition). The stimuli in Experiment 1 were shown on a quadratic display (20 visual degrees in width and height) as we aimed to use a display that was free of any spatial biases. In Experiment 2, participants always sat opposite of each other (to avoid any biases due to the seating arrangement) but stimuli were either shown on a display with a larger width than height (20 visual degrees wide and 10 visual degrees high-"Landscape" condition) or larger height than width (10 visual degrees wide and 20 visual degrees high"Portrait" condition). Note, the longer width and height for these displays was the same as the quadratic display's dimensions.

The experiment was structured in four blocks composed of 40 trials. Each set of two blocks contained one block, in which the selected condition is performed and one block, in which the assigned condition is performed. For the assigned condition, the 40 trials were composed of 20 left and right labor division trials and 20 top and bottom labor division trials. Across these two sets, depending on the experiment, we either changed the seating positions (Experiment 1) of co-actors or the shape of the screen display (Experiment 2). The order of all conditions was counterbalanced across pairs. Prior to the start of each block, participants were familiarized with the task procedure of the next block by the experimenter, who walked them through an example trial. Participants were instructed to not communicate with each other throughout the experiment. Along with assessing the participants' demographic data at the end of the experiment, participants were also required to rate how well they knew the other participant on a scale 1-5 $(1=$ not at all, $5=$ very well). We averaged these ratings across participants for both

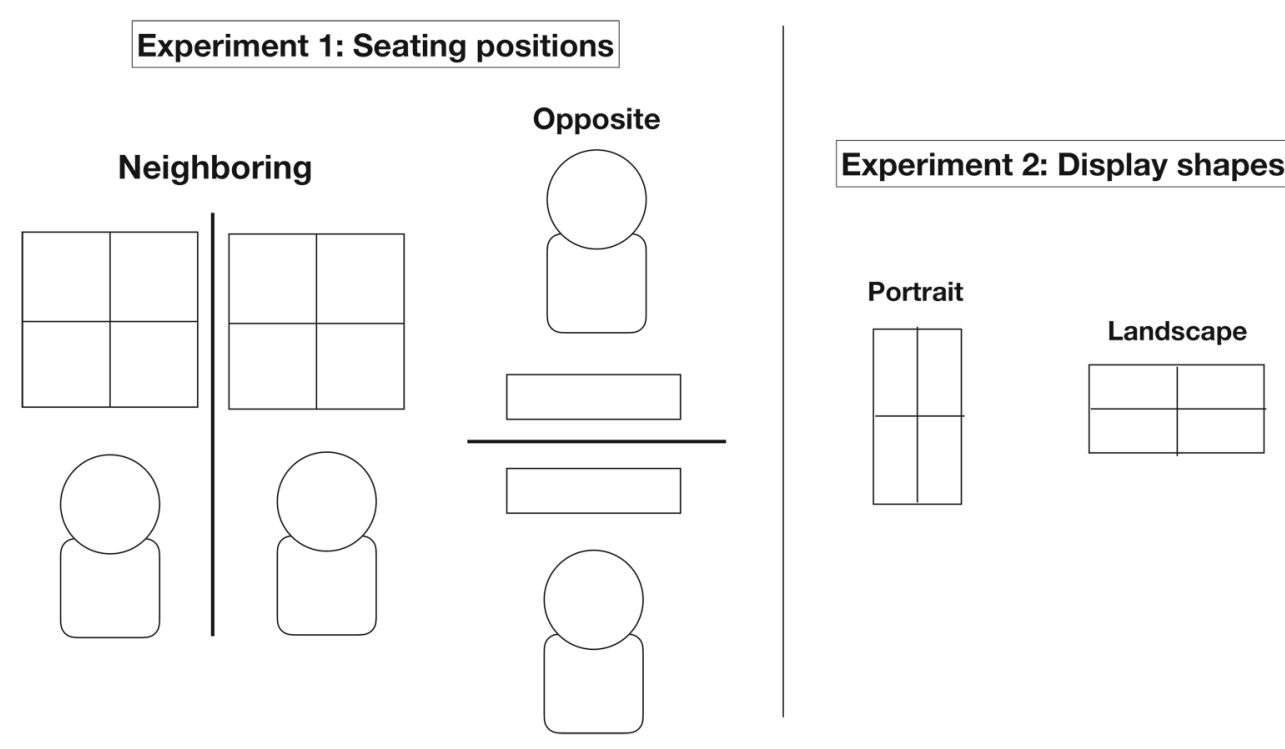

Fig. 3 Overview of experimental manipulation within each experiment. In Experiment 1, participants either sat next to each other or opposite of each other (left panel). In Experiment 2, participants performed the MOT task either on a portrait or landscape display (right panel). 
experiments and they were very low (Experiment 1: $M=$ $1.34, S D=1.00$; Experiment $2: M=1.69, S D=1.38)$, suggesting that pair members did not know each other. The experiment was programmed in Python 2.7.3 and took about 1 hour to complete.

\section{Results}

\section{Experiment 1: seating position}

To test whether we could replicate the benefit of tracking targets in both visual fields (Alvarez \& Cavanagh, 2005), we used a four factorial ANOVA only on the performance data of the assigned condition. The within-subject factors of the ANOVA were the Labor Division (left and right, top and bottom) and the Seating Position (Neighboring, Opposite). As between-subject factors, we included the Seating Position Order (Neighboring first, Neighboring second) and Condition Order (Assigned first, Assigned second). The dependent variable was the number of points each of the participants scored in the MOT task (for a descriptive overview, see Fig. 4a). To assure that the assumption of statistical independence is fulfilled, we averaged across the mean performance points of each co-actor within a pair. Generally, prior to applying all ANOVAs reported in the results section, we tested the statistical assumptions of normality using a Shapiro-Wilk test and sphericity using Mauchly's test for sphericity (if applicable). In case of violations of normality, we also performed pairwise comparisons using non-parametric tests (i.e., the Wilcoxon-Pratt signed-rank test for within-subject factors and the Mann-Whitney $U$ test for between-subject factors) and applied the Greenhouse Geisser correction to the degrees of freedom for sphericity violations.
We find a significant main effect of Labor Division $(F(1,12)=22.54, p<.001)$ but no interaction effects involving this factor $(p s>.440)$ and also no other significant effects $(p s>.066)$. As the normality assumption of the ANOVA was violated, we also compared the factor levels of the Labor Division factor using a WilcoxonPratt signed-rank test and found a significant difference for this comparison $(z=3.44, p<.001)$. In sum, our findings suggest that we replicate the findings by Alvarez and Cavanagh (2005).

To test which type of labor division participants chose in the selected condition, we performed an ANOVA with the same factors as above with the dependent variable the fraction of the selected labor division (i.e., trials in which a certain labor division was selected divided by the total number of trials in a respective condition). For a descriptive overview, see Fig. 4b. With regard to the factor Labor Division, note that pairs also selected a "diagonal" labor division (e.g., one participant tracked targets in the upper left and lower right quadrant while the other participant tracked objects in the other two quadrants) on a small number of trials. Hence, the factor Labor Division in the ANOVA now contains three levels (diagonal, left and right, top and bottom). We found a significant main effect of Labor Division $(F(1.23,14.77)=71.77, p<.001)$ but no other significant effects $(p s>.146)$. We followed up the main effect of Labor Division by comparing the proportions of the left and right and top and bottom divisions, and found a significant difference $(z=3.52, p<.001)$. To further investigate the absence of a Seating Position effect, we calculated the difference in proportions between the left and right, and top and bottom labor divisions for each seating position and subtracted these difference scores from each other for each pair. We computed a Bayes factor for these difference scores and found that the null hypothesis (i.e.,

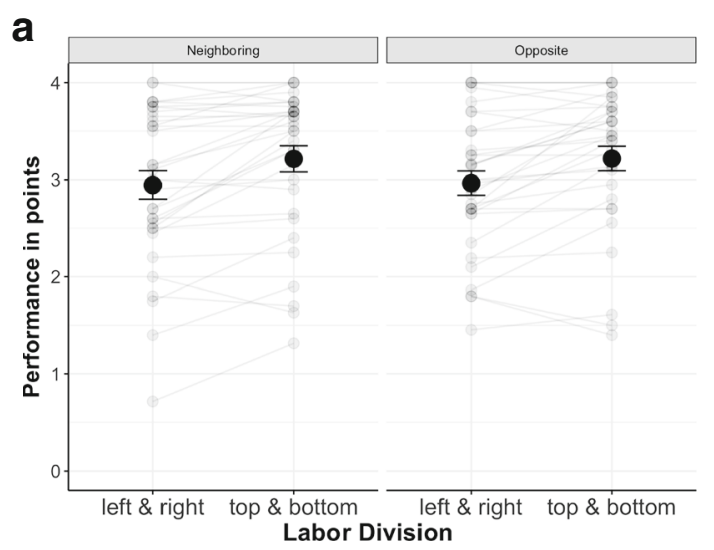

Fig. 4 Experiment 1: Results. a Individual performance (in points) in assigned condition as a function of labor division (left and right vs. top and bottom), separately for the Neighboring (left column) and Opposite (right column) seating positions. b Proportions of the selected labor

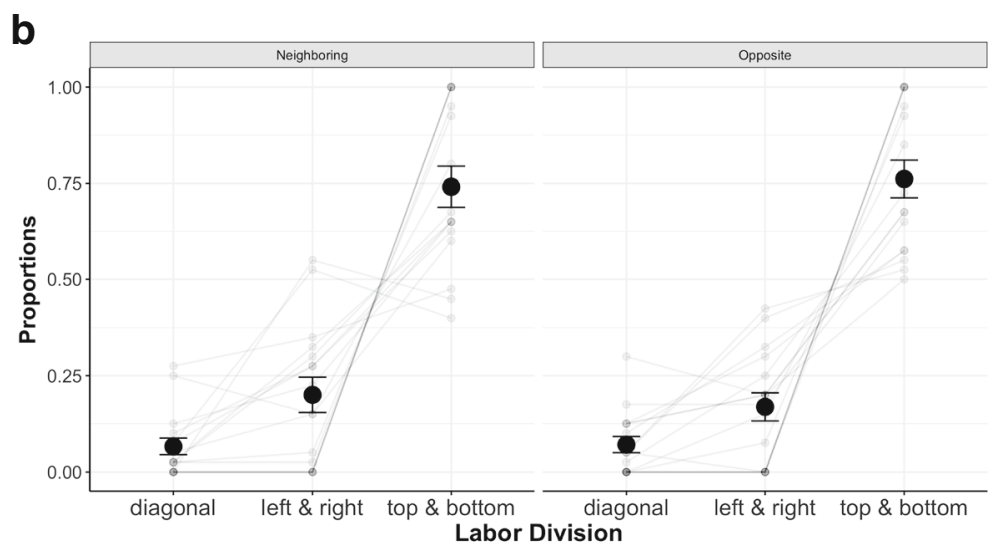

division as a function of labor division (diagonal, left and right, vs. top and bottom), separately for the Neighboring (left column) and Opposite (right column) seating positions. Error bars in all panels are standard error of the mean. 
that the selected division of labor is not affected by seating position) is 2.83 more likely than the alternative hypothesis (i.e., that the seating position influences the division of labor choice), suggesting that the seating arrangement does not affect the labor division choice.

We also investigated how these labor divisions develop over time. To do this we separated the 40 trials in the selected condition into segments with a length of five trials and calculated for each segment the fraction of each selected labor division. We extended the ANOVA above by the additional factor Trial Segment. We found a significant main effect of Labor Division $(F(2,24)=71.77, p<.001)$ and a significant two-way interaction effect between the factors Labor Division and Trial Segment $(F(2,24)=8.63, p<$ $.001)$. We also found a significant three-way interaction effect between the factors Labor Division, Trial Segment, and Condition Order $(F(2,24)=5.72, p=.009)$. To understand what is driving this three-way interaction, we plotted the proportions for all combinations of these three factors (see Fig. 5). One can see that a top and bottom labor division is favored early on for both levels of Condition Order. However, for pairs that performed the selected condition first, the preference for this division increased over time. In contrast, for pairs that performed the selected condition second, the top and bottom preference is already at its peak. In sum, the three-way interaction reflects the fact that a top and bottom labor division grows over time when the selected condition is performed first, and is already at its peak when the selected condition is performed second.

To follow up these analyses, we also tested how the participants' performance (averaged across labor divisions and pair members) changes over time for the different levels of the Condition Order factor (see Fig. 6, for a descriptive overview). We observe that the performances do not change over time. As labor division preferences are established early, performance seems to be steady throughout. We confirmed these observations by correlating the Time Segments variable with the performance variable, separately for each pair, and testing these correlations against zero. We find correlations do not differ significantly from zero (selected first: $t(8)=0.29, p=.777$; selected second: $t(8)=1.25, p=.252$ ). Calculating a Bayes factor for these comparisons, we find that the null hypothesis is more likely than the alternative hypothesis for both comparisons (selected first: 2.87; selected second: 1.64).

\section{Experiment 2: display orientation}

As above, we tested whether the benefit of tracking targets in both visual fields can be replicated, and again performed a four factorial ANOVA only on the data of the assigned condition. The two within-subjects factors of the ANOVA are the Labor Division (left and right, top and bottom) and Orientation (landscape, portrait). The betweensubject factors are the Orientation Order (Landscape first, Landscape second) and Condition Order (Assigned first, Assigned second). The dependent variable was again the number of scored points (for a descriptive overview, see Fig. 7). We find a significant main effect of Labor Division $(F(1,12)=19.70, p<.001)$ and a significant twoway interaction between the factors Labor Division and Orientation $(F(1,12)=41.16, p<.001)$. On top of that, we also find a significant three-way interaction between the factors Labor Division, Orientation, and Condition Order $(F(1,12)=7.19, p=.020)$ and a significant four-way interaction between all factors $(F(1,12)=6.38, p=.027)$.

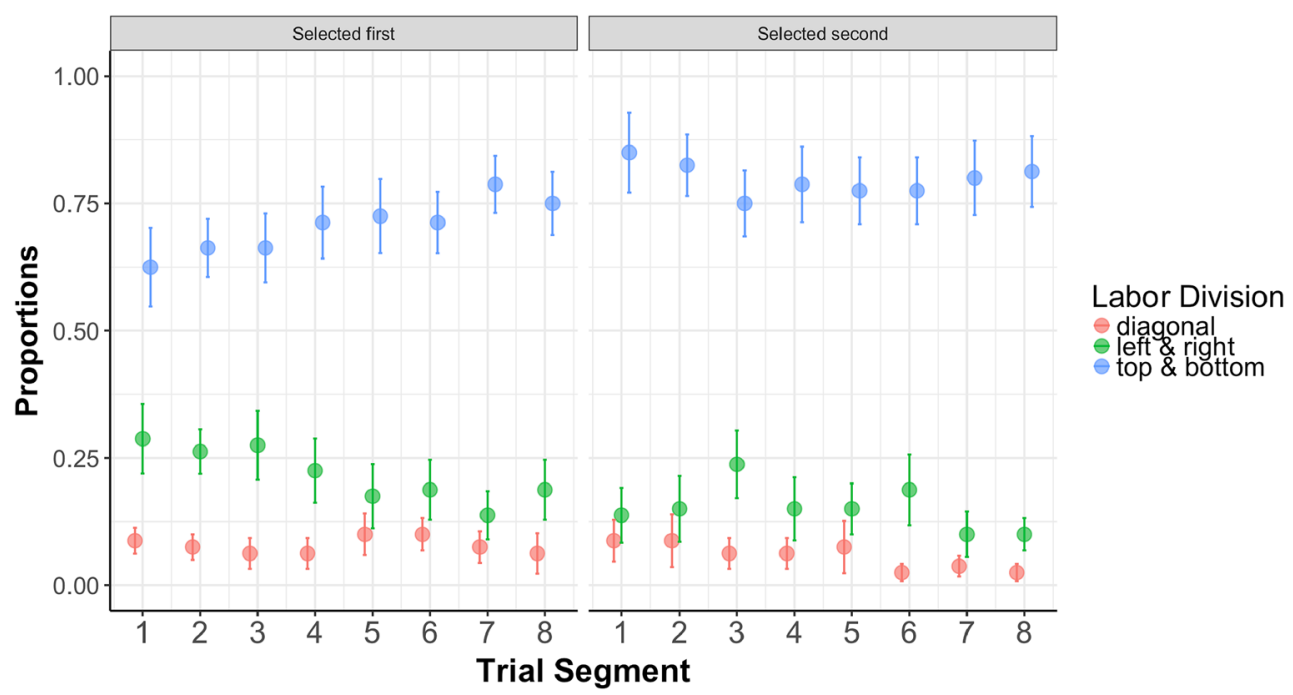

Fig. 5 Experiment 2: Proportions over time. Proportions of the selected labor division as a function of Trial Segment, separately for each labor division type (diagonal, left and right, \& top and bottom) and pairs performing the selected condition first (left column) or second (right column). Proportions calculated for each trial segment are based on five trials. Error bars in all panels are standard error of the mean. 


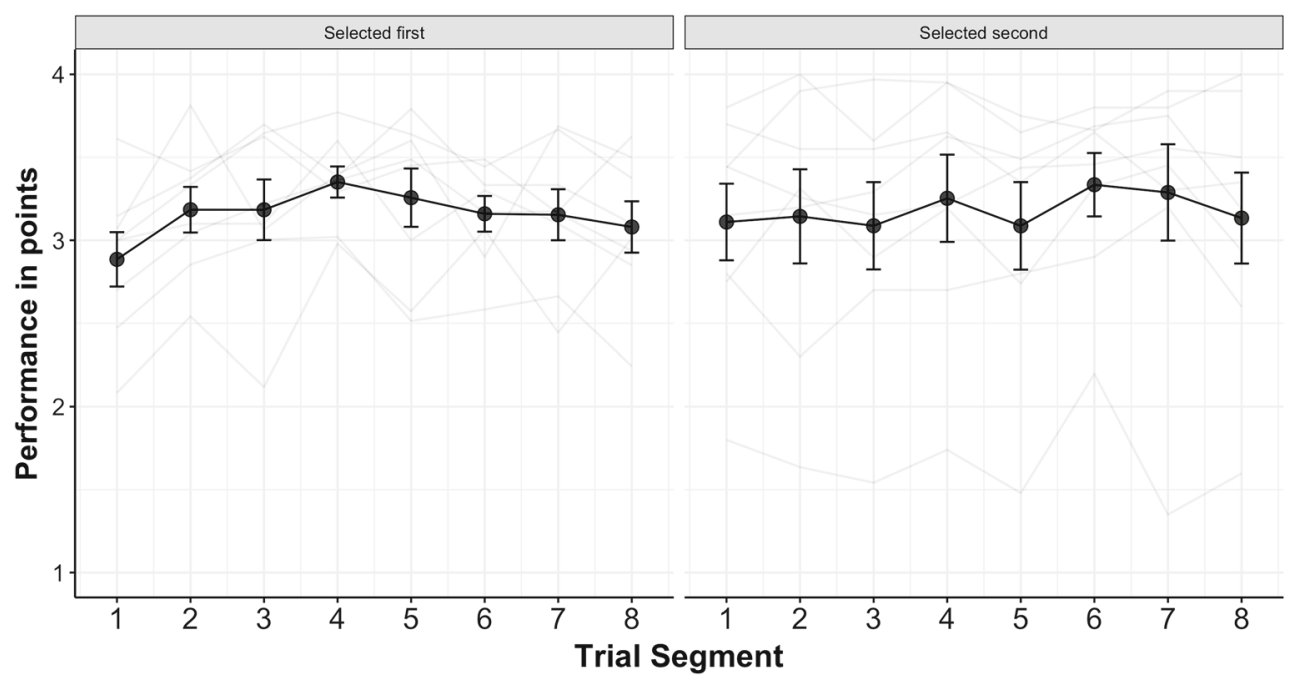

Fig. 6 Experiment 1: Performance over time. Performance as a function of Trial Segment, separately for pairs performing the selected condition first (left column) or second (right column). Performances calculated for each trial segment is based on five trials. Note, the performances are averaged across pair members and labor division types and displayed for the selected condition. Error bars in all panels are standard error of the mean.

To better understand these interactions, we computed the difference in performance between the levels of Labor Division (i.e., top and bottom performance minus left and right performance) for each combination of the remaining factors (see Fig. 8). Generally, we observe that the benefit of tracking across visual fields is present for the portrait display, but it is no longer present (or at least greatly reduced) for the landscape display. Moreover, the fourway interaction appears to be driven by participants that performed the tracking task first with a landscape display and the assigned condition second (i.e., after the selected condition). For this combination of factors, the difference between the landscape and portrait condition is even larger than for the other factor combinations.
Given that we find above that the benefit of tracking targets in both visual fields (relative to tracking targets in one visual field) is no longer present for the landscape display, we assessed which of the two following possibilities could explain this effect. One possibility is that for the landscape display there is no performance boost for tracking targets in both visual fields. That is, the benefit of tracking targets in both visual fields may be specific to certain shapes of displays (i.e., quadratic or portrait displays). The other possibility is that the landscape display allows for better performance when targets are tracked within only one visual field. In other words, the other stimuli displays negatively affected performance for tracking targets in one visual field while this is no longer the case for the landscape display. On

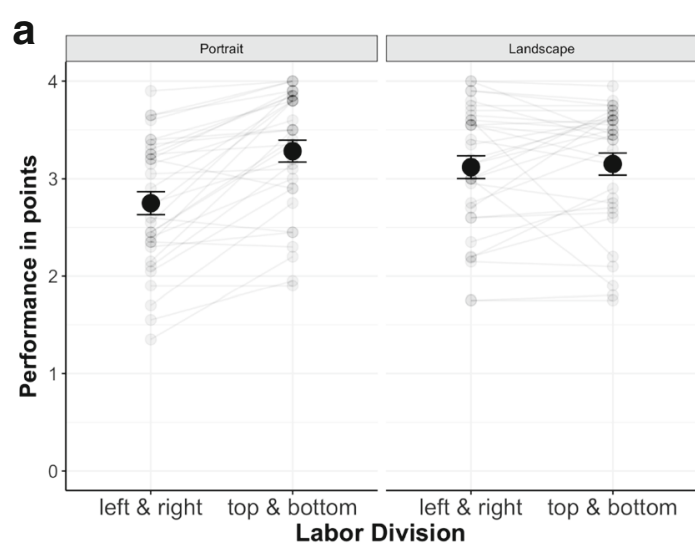

Fig. 7 Experiment 2: Results. a Individual performance (in points) in the assigned condition as a function of labor division, for the Portrait (left column) condition and Landscape (right column) condition. b Proportions of the selected labor division as a function of labor division

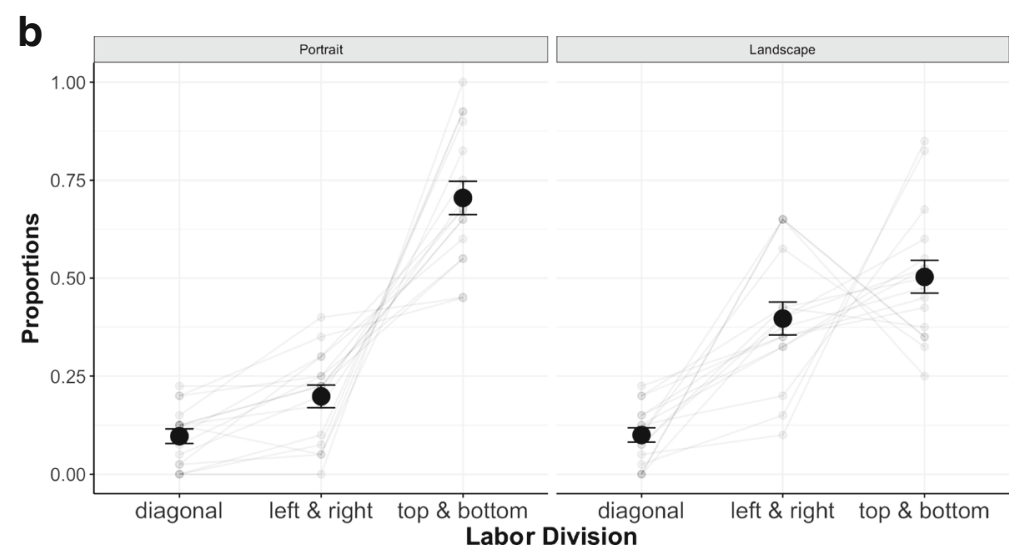

type (diagonal, left and right, \& top and bottom), for the Portrait (left column) condition and Landscape (right column) condition. Error bars in all panels are standard error of the mean. 


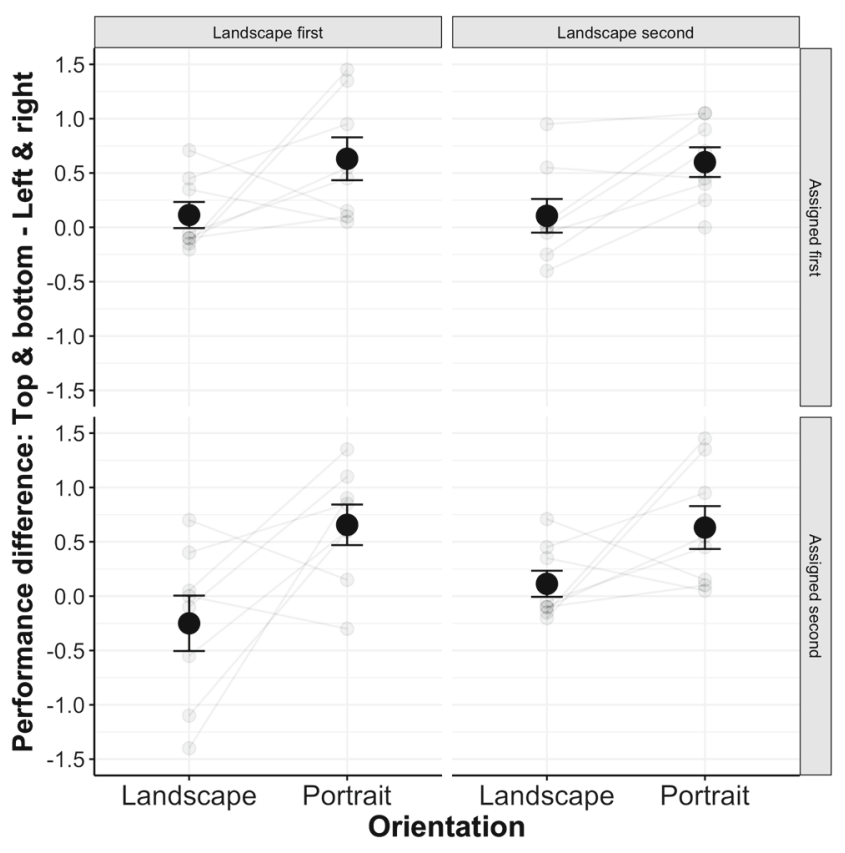

Fig. 8 Experiment 2: Four-way interaction. The difference in performance between a top and bottom and left and right labor division is plotted as a function of Orientation (landscape vs. portrait), for each combination of Condition Order (assigned first, assigned second) and Orientation Order (landscape first, landscape second). Error bars in all panels are standard error of the mean.

a descriptive level (see Fig. 7a), our data support the latter possibility, as regardless of which labor division is used, performance levels for the landscape display are as good as or better than performance for the portrait display. To support these observations, we compared the performance levels for the right and left labor division between displays and find that performance for the landscape display is significantly higher than for the portrait display $(t(31)=$ $4.14, p<.001)$, suggesting that tracking targets in one visual field is facilitated in the landscape display relative to the portrait display. In addition, we also compared the performances between the left and right labor division and top and bottom labor division for the landscape display and found no significant differences $(t(31)=0.37, p=.716)$. Calculating a Bayes factor for this latter comparison, we find that the null hypothesis (i.e., no differences between performances for the two labor divisions) is 3.85 more likely than the alternative hypothesis. In sum, our findings suggest that the landscape display facilitates tracking targets in one visual field.

To test which strategy was preferred by participants, we performed the same ANOVA as above with the dependent variable fraction of selected strategy. We find a significant main effect of Labor Division $(F(1.20,14.47)=57.64, p<$ $.001)$ and a significant interaction effect between the factors Labor Division and Orientation $(F(1.22,14.62)=16.08$, $p<.001)$. We followed up on this interaction effect by calculating the difference in proportions between the left and right, and top and bottom labor divisions, separately for the landscape and portrait displays. We then compared these residual scores and found them to be significantly different $(t(15)=4.69, p<.001)$. These results suggest that the preference for a top and bottom labor division is considerably reduced for the landscape display compared to the portrait display.

As for Experiment 1, we again investigated how these preferences develop over time using the same analytical approach. Thus, we extended the ANOVA by the additional factor Trial Segment. However, note that the factor Labor Division now only includes two levels, as the diagonal labor division was not chosen at all for a number of pairs in Experiment 2 (i.e., the data for this factor level is nearly a constant, which results in an error when applying the ANOVA). We find a significant main effect of Labor Division $(F(1,12)=25.44, p<.001)$ and a significant interaction effect $(F(1,12)=17.85, p=.001)$. In addition, we find a significant main effect of Trial Segment $(F(1,12)$ $=5.28, p=.040)$. However, we do not find any interaction effects involving the factors Trial Segment and Labor Division (all ps < .106). These findings suggest that the preference for a particular labor division is established early on and does not change over time (see Fig. 9, for a descriptive overview).

To follow up these analyses, we again tested how the participants' performance (averaged across labor divisions and pair members) changes over time (see Fig. 10, for a descriptive overview). Performance did not change over time as labor division preferences are established early on. We confirmed these observations by correlating the Time Segments variable with the performance variable, separately for each pair and level of Display Orientation. The correlations do not differ significantly from zero (Landscape display: $t(15)=0.30, p=.771$ ); Portrait display: $t(15)=0.34, p=.738)$. Calculating a Bayes factor for these comparisons, we find that the null hypothesis is 3.71 (for the landscape display) and 3.77 (for the portrait display) more likely than the alternative hypothesis.

\section{Discussion}

In the present study we aimed to replicate earlier findings by Alvarez and Cavanagh (2005) in a MOT task and test whether the pairs' labor division preference depends on either the seating arrangement (Experiment 1) or the shape of the task display (Experiment 2). We replicated earlier effects, finding that participants performance was higher when four target objects were tracked in both visual fields compared to only one visual field. These results suggest that earlier findings are robust, as our experimental design 


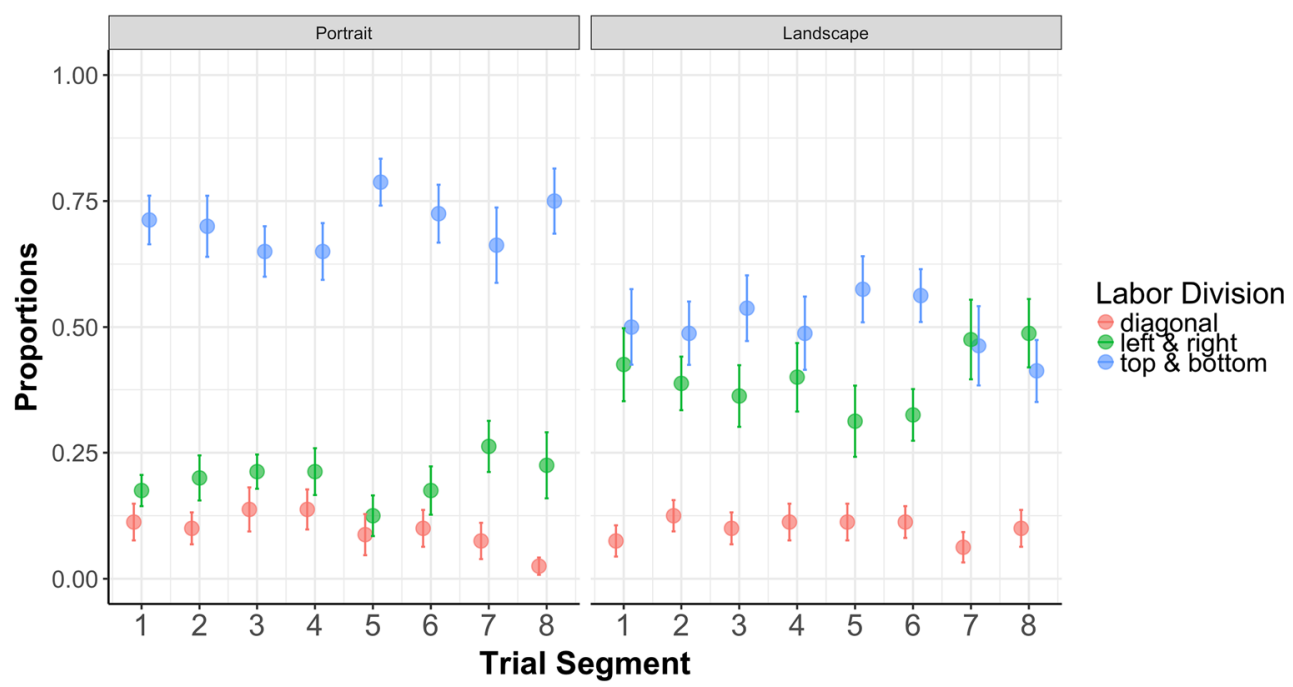

Fig. 9 Experiment 2: Proportions over time. Proportions of the selected labor division as a function of Trial Segment, separately for each labor division type (diagonal, left and right, \& top and bottom) and display orientation (Portrait (left column) and Landscape (right column)). Proportions calculated for each trial segment are based on five trials. Error bars in all panels are standard error of the mean.

involved pairs of participants whereas the original (Alvarez \& Cavanagh, 2005) study, and subsequent work based on their investigation (e.g., Störmer et al. (2014)), involved the performance of individuals working individually and in isolation.

When pair members could freely choose the labor division, we found they also preferred a top and bottom labor division-a division, which makes use of the benefit of tracking across left and right visual fields. This preferred division of labor was not influenced by the seating arrangement (i.e., sitting next to each other or opposite of each other; Experiment 1). Critically, when varying the shape of the display, the benefit of tracking targets in both visual fields was no longer present when the MOT task was performed in a landscape display while the benefit was still present for a portrait display. Convergent with these latter results, we also found that for the landscape display, pairs' preference for a top and bottom labor division was considerably reduced while the top and bottom preference was clearly present for the portrait display. Taken together, our findings suggest that participants' strategy preference is influenced by the availability of attentional capacity. That is, if tracking targets in both visual fields results in a higher tracking ability, participants preferentially choose a top and

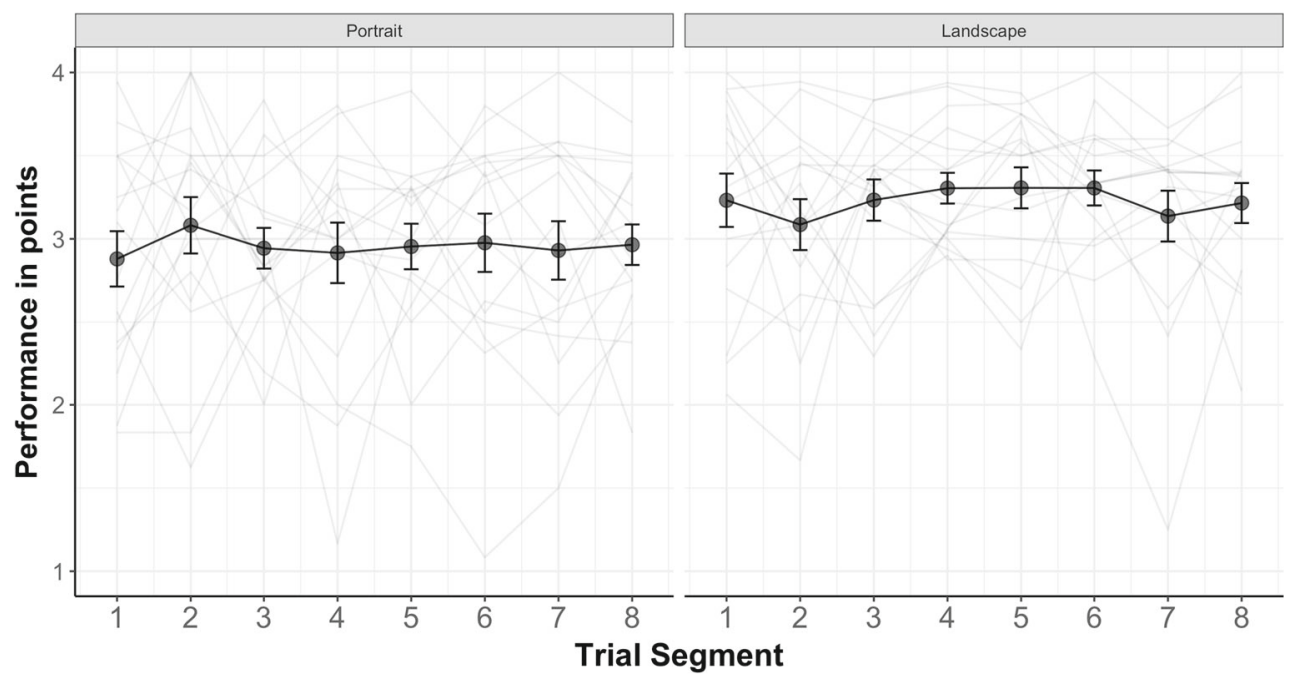

Fig. 10 Experiment 2: Performance over time. Performance as a function of Trial Segment, separately for Portrait (left column) and Landscape (right column) orientation. Performance for each trial segment is based on five trials. Note, the performances are averaged across pair members and labor division types and displayed for the selected condition. Error bars in all panels are standard error of the mean. 
bottom labor division that takes advantage of this benefit. However, if there is no such benefit present as with the landscape display, participants have no clear labor division preference.

Given earlier results by Alvarez and Cavanagh (2005), our findings suggest that the benefit of tracking targets in both visual fields relative to tracking only in one visual field is influenced by the shape of the display in which the MOT task is performed. To explain these results, we want to point out how narrowing the display in one of the dimensions has most likely changed the MOT task demands in two key ways. First, by narrowing the display in one dimension we changed the proximity between objects within a quadrant in that dimension (as they now move in a smaller space), which likely increases the demands made on the spatial acuity of attention (He et al., 1997; Intriligator \& Cavanagh, 2001) and in turn makes the task more difficult. Second and perhaps more importantly, the distances between target objects were systematically larger in one dimension than in the other dimension, depending on the display's orientation. For the landscape display, targets objects were further apart in the horizontal dimension whereas for the portrait display target objects were further apart in the vertical dimension. We suggest that what drives the benefit of tracking across visual fields is the relative difficulty to track objects within one visual field if they are spaced far apart in the vertical dimension. If the display is a landscape display, the vertical distance between target objects is reduced considerably and hence the source of the difficulty for tracking targets in one visual field is reduced as well. Supporting these observations, we find that for the landscape display tracking performance is highly similar between a left and right division, and a top and bottom division. That is, there is no clear benefit for tracking targets across visual fields. In sum, our findings suggest that the benefit of tracking targets across visual fields is attributable to a difficulty in tracking targets in one visual field, and that this difficulty is absent for a landscape display, possibly because the vertical distance between targets is reduced.

Discussing our results with regard to our earlier study on a joint MOT task (Wahn et al., 2017), the present findings suggest that participants in our earlier study most likely had preferred a left and right labor division due to the landscape display and not due to the seating arrangement. That is, only our manipulation of the shape of the task display resulted in a change of the labor division preference while the seating arrangement manipulation had no effect. Moreover, as noted above, the manipulation of the task display shape also affected task performance, resulting in comparable performance levels between a top and bottom and left and right labor division for a landscape display. In sum, if tracking objects in two visual fields is clearly beneficial (as for the quadratic or portrait display), then humans prefer a top and bottom labor division. If neither tracking in both visual fields nor only one visual field is more beneficial (as for the landscape display), the preference for a top and bottom division is greatly reduced. Given these results, we propose that co-actors are sensitive to changes within their environment which allows them to choose a labor division that maximizes use of their individual attentional capacities in joint spatial tasks. To follow-up on this proposal, future studies could test whether the present findings also generalize to other joint spatial tasks such as, for instance, joint visual search (Brennan et al., 2008; Brennan \& Enns, 2015; Szymanski et al., 2017; Wahn et al., 2016b; Wahn et al., 2018a; Wahn et al., 2020).

Acknowledgments We acknowledge the support of a DFG research fellowship (WA 4153/2-1) awarded to BW, and an NSERC Discovery Grant to AK.

Open Practices Statement All data of the present experiments are available in the following repository: https://osf.io/xs8az/?view only $=05926604902343 \mathrm{a} 19618 \mathrm{abfc} 2 \mathrm{~b} 8 \mathrm{c} 9 \mathrm{~b} 7 \mathrm{~b}$.

\section{References}

Alnæs, D., Sneve, M. H., Espeseth, T., Endestad, T., van de Pavert, S. H. P., \& Laeng, B. (2014). Pupil size signals mental effort deployed during multiple object tracking and predicts brain activity in the dorsal attention network and the locus coeruleus. Journal of Vision, 14(4), 1-1.

Alvarez, G. A., \& Cavanagh, P. (2005). Independent resources for attentional tracking in the left and right visual hemifields. Psychological Science, 16(8), 637-643.

Alvarez, G. A., \& Franconeri, S. L. (2007). How many objects can you track?: Evidence for a resource-limited attentive tracking mechanism. Journal of Vision, 7(13), 14-14.

Brennan, A. A., \& Enns, J. T. (2015). When two heads are better than one: Interactive versus independent benefits of collaborative cognition. Psychonomic Bulletina and Review, 22(4), 1076-1082.

Brennan, S. E., Chen, X., Dickinson, C. A., Neider, M. B., \& Zelinsky, G. J. (2008). Coordinating cognition: The costs and benefits of shared gaze during collaborative search. Cognition, 106(3), $1465-1477$.

Cavanagh, P., \& Alvarez, G. A. (2005). Tracking multiple targets with multifocal attention. Trends in Cognitive Sciences, 9(7), 349-354.

Chen, W.-Y., Howe, P. D., \& Holcombe, A. O. (2013). Resource demands of object tracking and differential allocation of the resource. Attention, Perception, and Psychophysics, 75(4), 710725 .

Chun, M. M., Golomb, J. D., \& Turk-Browne, N. B. (2011). A taxonomy of external and internal attention. Annual Review of Psychology, 62, 73-101.

Goldman, A., \& de Vignemont, F. (2009). Is social cognition embodied?. Trends in Cognitive Sciences, 13(4), 154-159.

He, S., Cavanagh, P., \& Intriligator, J. (1997). Attentional resolution. Trends in Cognitive Sciences, 1(3), 115-121.

Intriligator, J., \& Cavanagh, P. (2001). The spatial resolution of visual attention. Cognitive Psychology, 43(3), 171-216.

James, W. (1890). The principles of psychology. Cambridge: Harvard UP.

Lavie, N. (2005). Distracted and confused?: Selective attention under load. Trends in Cognitive Sciences, 9(2), 75-82. 
Marois, R., \& Ivanoff, J. (2005). Capacity limits of information processing in the brain. Trends in Cognitive Sciences, 9(6), 296305.

Meier, B. P., Schnall, S., Schwarz, N., \& Bargh, J. A. (2012). Embodiment in social psychology. Topics in Cognitive Science, 4(4), 705-716.

Pylyshyn, Z. W., \& Storm, R. W. (1988). Tracking multiple independent targets: Evidence for a parallel tracking mechanism. Spatial Vision, 3(3), 179-197.

Scholl, B. J. (2009). What have we learned about attention from multiple object tracking (and vice versa). Computation, Cognition, and Pylyshyn, 49-78.

Sebanz, N., Bekkering, H., \& Knoblich, G. (2006). Joint action: Bodies and minds moving together. Trends in Cognitive Sciences, 10(2), 70-76.

Smith, E. R., \& Semin, G. R. (2007). Situated social cognition. Current Directions in Psychological Science, 16(3), 132-135.

Störmer, V. S., Alvarez, G. A., \& Cavanagh, P. (2014). Withinhemifield competition in early visual areas limits the ability to track multiple objects with attention. Journal of Neuroscience, 34(35), 11526-11533.

Szymanski, C., Pesquita, A., Brennan, A. A., Perdikis, D., Enns, J. T., Brick, T. R., ..., Lindenberger, U. (2017). Teams on the same wavelength perform better: Inter-brain phase synchronization constitutes a neural substrate for social facilitation. Neuroimage, $152,425-436$

Vesper, C., Abramova, E., Bütepage, J., Ciardo, F., Crossey, B., Effenberg, A., ..., Wahn, B. (2017). Joint action: Mental representations, shared information and general mechanisms for coordinating with others. Frontiers in Psychology, 7, 2039.
Wahn, B., Czeszumski, A., \& König, P. (2018a). Performance similarities predict collective benefits in dyadic and triadic joint visual search. PLoS ONE, 13(1), e0191179.

Wahn, B., Czeszumski, A., Labusch, M., Kingstone, A., \& König, P. (2020). Dyadic and triadic search: Benefits, costs, and predictors of group performance. Attention, Perception, \& Psychophysics, $1-19$.

Wahn, B., Ferris, D. P., Hairston, W. D., \& König, P. (2016a). Pupil sizes scale with attentional load and task experience in a multiple object tracking task. PloS One, 11(12), e0168087.

Wahn, B., Kingstone, A., \& König, P. (2017). Two trackers are better than one: information about the co-actor's actions and performance scores contribute to the collective benefit in a joint visuospatial task. Frontiers in Psychology, 8, 669.

Wahn, B., Kingstone, A., \& König, P. (2018b). Group benefits in joint perceptual tasks: A review. Annals of the New York Academy of Sciences, 1426(1), 166-178.

Wahn, B., \& König, P. (2017a). Can limitations of visuospatial attention be circumvented? A review. Frontiers in Psychology, 8 , 1896.

Wahn, B., \& König, P. (2017b). Is attentional resource allocation across sensory modalities task-dependent?. Advances in Cognitive Psychology, 13(1), 83.

Wahn, B., Schwandt, J., Krüger, M., Crafa, D., Nunnendorf, V., \& König, P. (2016b). Multisensory teamwork: Using a tactile or an auditory display to exchange gaze information improves performance in joint visual search. Ergonomics, 59(6), 781-795.

Publisher's note Springer Nature remains neutral with regard to jurisdictional claims in published maps and institutional affiliations. 